\title{
Comparative evaluation for wound healing potentials of bulk and nano forms of zinc oxide ointment
}

\author{
V. Gupta ${ }^{1 *}$, P. K. Verma ${ }^{2}$, A. Gupta ${ }^{3}$, V. Kant ${ }^{4}$, P. Kumar ${ }^{5}$, M. Sharma ${ }^{1}$ \\ ${ }^{1}$ Department of Chemistry, University of Jammu, India \\ ${ }^{2}$ Division of Pharmacology \& Toxicology, SKUAST-Jammu, J\&K, India \\ ${ }^{3}$ Department of Physics, University of Jammu, J\&K, India \\ ${ }^{4}$ Division of Pharmacology \& Toxicology, IVRI, Bareilly, UP, India; \\ Presently: Department of Vety Pharmacology \& Toxicology, LUVAS, Hisar, India \\ ${ }^{5}$ Department of Pathology, IVRI, Bareilly, UP, India \\ *vijayta1gupta@gmail.com
}

DOI 10.17586/2220-8054-2020-11-4-453-461

\begin{abstract}
Development of nanotechnology has led to massive breakthroughs in the area of wound healing. Recently, metal oxide nanoparticles have shown a broad range of applications in biomedical fields. The lack of potent healing agents for complicated wounds and healing potentials of zinc oxide $(\mathrm{ZnO})$ motivated us to evaluate the wound healing potentials of nano $\mathrm{ZnO}$ in comparison to its bulk form in rats. In the present study, single open excision wounds $\left(2 \times 2 \mathrm{~cm}^{2}\right)$ were created on the backs of fifteen rats and divided into Group I, II and III. On the wounds of group I, II and III, topical application of ointment base, bulk $\mathrm{ZnO}(20 \%)$ and $\mathrm{ZnO}$ nanoparticles (2\%) was done for 14 days, respectively. Significantly smaller wound area and increased percent wound contraction was evident in the $\mathrm{ZnO}$ nanoparticles-treated group. Histopathological analysis revealed that the $\mathrm{ZnO}$ nanoparticle-treated wounds possessed reduced numbers of fibroblasts and blood vessels. However, collagen fibers in $\mathrm{ZnO}$ treated group were compactly arranged in thick bundles with a well-organized manner and orientation. The newly formed epithelial layer was also covering more area of healing tissue in the $\mathrm{ZnO}$ nanoparticle-treated group. The $\mathrm{ZnO}$ nanoparticle-treated group also revealed the higher overall wound maturity score, as compared to other groups. In view of this, it might be concluded that topical application of $\mathrm{ZnO}$ nanoparticles ( $2 \%$ ) caused faster wound healing and the healing was better than bulk $\mathrm{ZnO}$ treatment, even at ten-fold lower concentration.
\end{abstract}

Keywords: Zinc oxide, nanoparticles, wound healing, collagen, epithelial layer, rats.

Received: 19 April 2020

Revised: 1 May 2020

\section{Introduction}

During the last few years, nanotechnology has become an emergent interdisciplinary field of research by combining the material science, bio-nanoscience, and technology. It has been considered as the technological innovation of the $21^{\text {st }}$ century. Nanotechnology involves the design, characterization, synthesis and application of different materials, structures, devices and systems by controlling their shape as well as size at the nanometer scale. Research and development in this field at various levels are increasing rapidly. Engineered nanoparticles are key constituents of the nanotechnology industry and number of consumer products containing nanoparticles is rapidly increasing. Nanoparticles have greater potential to cross biological barriers to reach pulmonary connective tissues, lymphatics, blood circulation and critical organs in comparison with micron-sized particles [1-3]. Their smaller size and large surface area also enhance their ability to enter into cells and become more biologically active [4-6]. Nanotechnology has shown tremendous broad application in many fields, including medicine. This technology has become the forefront of research and possesses tremendous potential to revolutionize the medical sector.

Currently, impaired wound healing in humans and animals has become a big challenge in medical and veterinary fields. Although wound healing is a natural phenomenon, the conversion of an acute wound to a chronic wound can occur due to the persistence of chronic conditions (diabetes mellitus, venous stasis ulcers, skin ulcers etc.) resulting in difficulties for wound management. The prevalence of patients with chronic wounds has increased sharply in recent years. The probabilities of occurring of cutaneous wounds are more, as skin is vulnerable to a variety of external factors and resulting in different types of skin damage. The available traditional therapies generally involve high cost and long-lasting treatments with an ulcer relapse rate of above $70 \%$ [7]. Significant efforts have been made towards wound care management in recent years for framing the new therapeutic approaches and technologies, however,satisfactory results have still not been achieved. The innovative and impressive development of nanotechnology has led to massive growth in the direction of nano-drug delivery systems for wound healing and skin regeneration. Additionally, nanoparticles of metals and their oxides have emerged in recent years with a broad range of applications in biomedical field 
including antibacterial, anticancer drug/gene delivery, cell imaging, biosensing, transdermal antibiotic patches, cosmetics etc. [8]. It has been observed that nanoparticle of metals and their oxides can exhibit unique functions, which are not observed in bulk phases. In recent years, some studies have revealed the wound healing of many compounds, including metals and their oxides. Application of silver nanoparticles to wounds showed that healing times were less when compared to another standard drug (Povidone-iodine) [9]. Copper nanoparticles have shown acceleration of wound healing via promotion of skin cell migration, proliferation, and neovascularization [19]. Copper nanoparticleembedded hydrogels have also displayed promising potential for skin tissue regeneration [11]. Thrombin-conjugated iron oxide nanoparticles have caused the enhancement of incisional wound healing [12]. Recently, curcumin nanoformulation enhanced wound repair by inhibiting the inflammatory response, stimulating angiogenesis, inducing fibroblast proliferation as well as enhancing reepithelization and collagen synthesis [13].

Zinc $(\mathrm{Zn})$ is one of the most abundant metallic elements in the body after iron. Zinc regulates DNA synthesis, normal growth, brain development, behavioral responses, reproduction, membrane stability, bone formation, wound healing etc. [14]. Zinc oxide ( $\mathrm{ZnO})$ is widely used in several products such as paints, coatings and cosmetic products $[15,16]$. ZnO has been declared as "Generally Recognized as Safe" (GRAS) by the US Food and Drug Administration (21, CFR 182, 8991). In zinc-deficient rats, reduced granulation tissue deposition, decreased tensile strengths and delayed wound closure rates have been observed [17,18]. Zinc has shown to accelerate the wound healing process when applied on open wounds [19]. Additionally, zinc administered topically appears to be beneficial for healing than given orally [20]. Recently, nano $\mathrm{ZnO}$ has also been widely researched as potent compound for biological applications due to its excellent antimicrobial, anti-inflammatory and angiogenic properties [21]. ZnO nanoparticles are also considered comparatively inexpensive and somewhat less toxic than other metal oxide nanoparticles. To the best of our knowledge, comparative wound healing studies of bulk and nano $\mathrm{ZnO}$ are very limited to claim the better potential of nanformulation of $\mathrm{ZnO}$. In view of this, the present study was planned to evaluate the wound healing potential of $\mathrm{ZnO}$ nanoparticles in comparison to its bulk form.

\section{Materials and method}

\subsection{Chemicals used}

All the chemicals used in this study were of analytical grade and used as received without further purification. Zinc acetate and sodium hydroxide were used for the preparation of $\mathrm{ZnO}$ nanoparticles, and were purchased from Sigma Aldrich, USA. The ZnO extrapure (99.5 \%; Catalogue number: 67062) of analytical grade was purchased from Sisco Research Laboratories (SRL), New Delhi, India.Other chemicals like formaldehyde, acetone, acetic acid, ethanol, white petroleum jelly, xylene etcwere also purchased from SRL. Hematoxylin stain, Eosin stain, Biebrich scarlet stain, phosphomolybdic acid, aniline blue etc. were purchased from Himedia, Mumbai, India.

\subsection{Synthesis and characterization of $\mathrm{ZnO}$ nanoparticles}

Zinc acetate $\left[\mathrm{Zn}\left(\mathrm{CH}_{3} \mathrm{COO}\right)_{2} \cdot 2 \mathrm{H}_{2} \mathrm{O}\right]$ and sodium hydroxide were the precursors used in the synthesis of $\mathrm{ZnO}$ nanoparticles. The $\mathrm{ZnO}$ nanoparticles were prepared as per the method described by Rezende et al. [22]. Briefly, an aqueous solution of zinc acetate $(0.5 \mathrm{M})$ was kept on magnetic stirrer at $80^{\circ} \mathrm{C}$ for one hour for complete dissolution. Thereafter, aqueous solution of $\mathrm{NaOH}(2.5 \mathrm{M})$ was added under high speed and constant stirring. The addition was dropwise (slowly for $45 \mathrm{~min}$ ) and touching the walls of the vessel till the $\mathrm{pH}$ reaches to 12 . The reaction was kept for $2 \mathrm{hrs}$ after complete addition of sodium hydroxide. After the completion of reaction, the solution was maintained overnight and the supernatant solution was separated carefully on next day. The remaining solution was centrifuged for $10 \mathrm{~min}$, and the precipitate was removed. Thus, precipitated $\mathrm{ZnO}$ nanoparticles were washed three times with triply distilled water and ethanol to remove the byproducts which were bound with the precipitate and then dried in oven at about $60{ }^{\circ} \mathrm{C}$. The white powder obtained is subjected to calcinations at $600{ }^{\circ} \mathrm{C}$ for 3 hours. Characterization of synthesized $\mathrm{ZnO}$ nanoparticles was carried out by using particle size analyzer (PSA), transmission electron microscopy (TEM) and scanning electron microscopy (SEM). The particle size distribution (PSD) analysis of the synthesized nanoparticles was done by using Malvern Instruments Zetasizer Nano-ZS instrument. The size and morphology of nanoparticles was determined by using Transmission Electron Microscope [Hitachi (H-7500) microscope] operating at $80 \mathrm{kV}$. The morphology of the particles was observed by a scanning electron microscope (SEM-EDS) using SEM (make JEOL Model JSM-6390LV).

\subsection{Preparation of ointments}

The ointment base was prepared by mixing the hard paraffin (5\%), soft paraffin (90\%) and lanolin (5\%) by the fusion method. The ointments of bulk $\mathrm{ZnO}(20 \%)$ and $\mathrm{ZnO}$ nanoparticles $(2 \%)$ were prepared in this ointment base 
by the incorporation method. The prepared ointment base, bulk $\mathrm{ZnO}$ ointment and ointment of $\mathrm{ZnO}$ nanoparticles were stored at $4{ }^{\circ} \mathrm{C}$ till further uses for wound healing studies.

\subsection{Cutaneous wound healing study}

\section{(a) Wound creation and grouping}

Fifteen healthy male Wistar rats $(140-160 \mathrm{~g} ; 8-10$ weeks of age $)$ were used in this study. The overnightfasted rats were anesthetized by intraperitoneal (i.p.) injection of ketamine $(50 \mathrm{mg} / \mathrm{kg}$, i.p. $)+x y l a z i n e ~(5 \mathrm{mg} / \mathrm{kg}$, i.p.) combination. After achieving the anesthesia, dorsal skin of the rats was shaved and cleaned with $70 \%$ ethanol. After this, a square shaped $2 \times 2 \mathrm{~cm}^{2}\left(\approx 400 \mathrm{~mm}^{2}\right)$ full thickness excision cutaneous wound was created on the back (thoraco-lumber) region. Each operated rat was housed separately in disinfected cages. Surgically wounded rats were equally divided in three groups (five each). Ad libitum access to feed and water, 12-hour light-dark cycle and adequate ventilation was provided to rats throughout the entire experiment. The temperature of the experimental room was maintained between $22-26 \circ \mathrm{C}$ during the whole experiment. The details of three groups are given below:

Group I (Control or ointment base-treated): Ointment base was applied topically once daily for 14 days. Group II (Bulk ZnO-treated): Bulk $\mathrm{ZnO}(20 \%)$ in ointment base was applied topically once daily for 14 days. Group III (Nano ZnO-treated): $\mathrm{ZnO}$ nanoparticles $(2 \%)$ in ointment base was applied topically once daily for 14 days.

The different ointments applied in above mentioned groups were just smeared on the wound area.

\section{(b) Photography of wounds and calculation of wound area as well as percent wound contraction}

Photography of each wound was done on days $0,3,7$ and 14 post-wounding to assess the gross changes in the wounds after different treatments. Each wound margins was traced on a transparent paper with the help of fine tip permanent marker. The area (in $\mathrm{mm}^{2}$ ) within the boundaries of each wound tracing was determined planimetrically. The wound area on 0 day of each animal was measured at a predetermined time interval starting at $3 \mathrm{~h}$ post-wounding. Thereafter, subsequent measurements of wound areas of all the groups were taken on day 3, 7 and 14 post-wounding. The result of wound measurements was expressed as absolute values and relative values or per cent wound contraction. The absolute values are actual measurements of wounds obtained at each given interval, whereas in relative values the wound contraction is expressed as per cent values of the 0-day measurements and was calculated by Wilson' formula as follows:

$$
\% \text { wound contraction }=\frac{0 \text { day wound area }- \text { wound area on perticular day }}{0 \text { day wound area }} \times 100 \text {. }
$$

\section{(c) Collection of tissue}

Healing tissue of each animal of all the groups was collected under intramuscular general anesthesia (xylazine + ketamine combination). A portion of collected tissue was immediately preserved in $10 \%$ formalin for histopathological studies.

\section{(d) Histopathology}

Hematoxylin and Eosin (H\&E) staining and scoring:

The collected formalin-fixed tissues were embedded in paraffin wax. Thereafter, approximately $6 \mu \mathrm{m}$ thick tissue sections were cut. The $\mathrm{H} \& \mathrm{E}$ staining of one set of tissue sections were done for each rat of all groups as per standard method. The stained sections were analyzed and scored under a light microscope at different magnifications. Scoring for the inflammatory cells [23], epithelialization [24] and overall wound maturity [25,26] was done as per the standard methods. Scoring for blood vessel density was done by counting the number of blood vessels in 35 random fields at higher magnification $(40 \times)$. The histopathological properties of different tissue sections and scoring were blindly done.

Masson's trichrome staining for collagen:

The evaluation of collagen synthesis in the healing tissues was determined by using the Masson's trichrome stain. Masson's trichrome staining of each tissue section was done as per standard method. The stained sections were analyzed under a light microscope at different magnifications. In this staining, nuclei stained black; cytoplasm, muscles and erythrocytes as red and collagen stained blue.

\subsection{Statistical analysis}

Data were analyzed by one and two way analysis of variance (ANOVA) followed by Bonferroni's post-test using the GraphPad Prism v4.03 software program (San Diego, CA, USA). The differences between the different treatment groups were considered statistically significant at $P \leq 0.05$ or lower. All data were expressed as mean \pm standard error of mean (S.E.M.) of five animals. 


\section{Results and discussion}

In our present investigation, the average size of chemically synthesized $\mathrm{ZnO}$ nanoparticles obsereved in Zetasizer was $125.64 \mathrm{~nm}$ with a polydispersity index of 0.424 . The TEM image of $\mathrm{ZnO}$ nanoparticles revealed average size of $70-115 \mathrm{~nm}$ and spherical shape of particles (Fig. 1a). The SEM analysis of the ZnO nanoparticles further revealed a uniform shape and size for $\mathrm{ZnO}$ nanoparticles and aggregations of chemically synthesized nanoparticles (Fig. 1b). It was also evident that nanoparticles were mainly in granules and well crystallized. Therefore, in our present study, the size of the $\mathrm{ZnO}$ particles was in the nanometer range. It has been well observed in earlier studies that small size of the nanoparticles allows their internalization into cells, and to interact with biomolecules within or on the cell surface, enabling them potentially to affect cellular responses in a dynamic and selective manner that makes them well suited for biological applications [27]. Additionally, the spherical shape of the nanoparticles is well suited for the efficient topical actions of the nanoparticles.
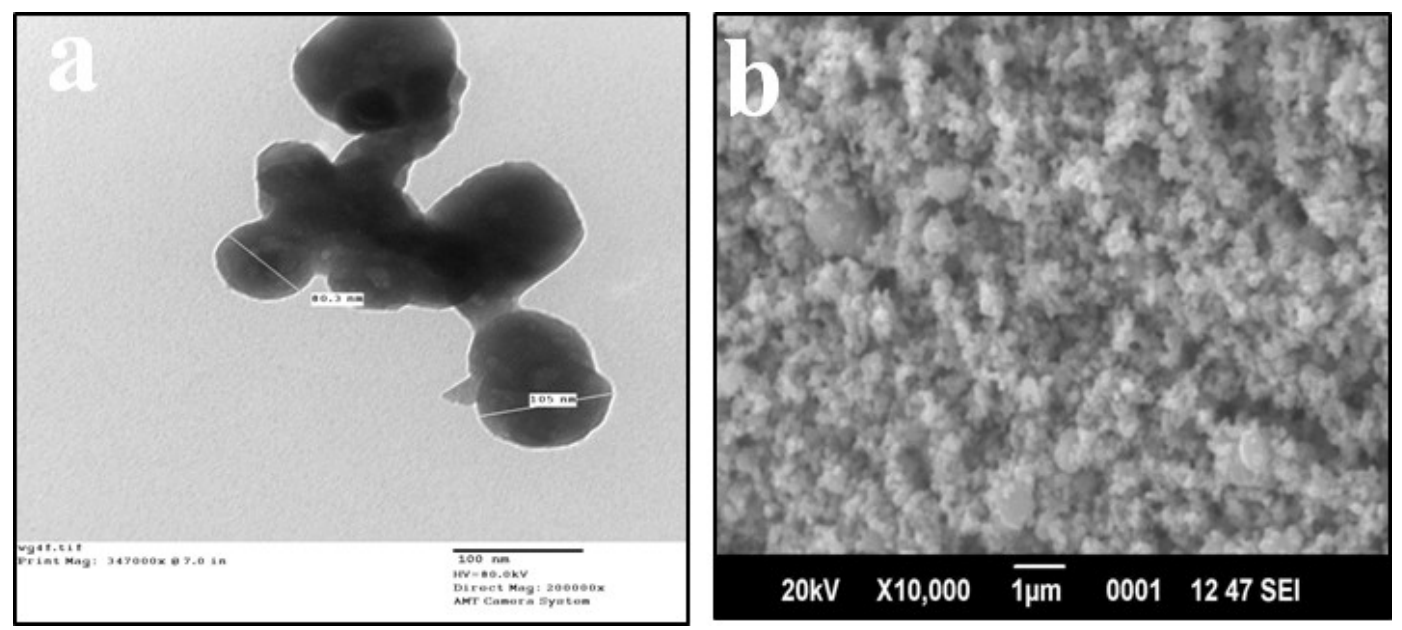

FIG. 1. Representative (a) TEM and (b) SEM image of $\mathrm{ZnO}$ nanoparticles

Cutaneous wound healing constitutes an important aspect in rehabilitation medicine. Wound healing has been considered as one of the most vexing problems over the globe. Wounds cause significant morbidity and mortality in humans and animals [28]. Numbers of patients have simple or complicated wounds are increasing day by day. Impairments in the wound healing progress sometimes lead to amputations [29]. Cutaneous wounds cause pain, discomfort, superficial and chronic infection, significant cost associated with the long term treatment. Different treatments available for wound healing are there which include antibiotics, occlusive layers, bandages, poultices and mechanical devices etc. All these treatments become less effective or ineffective for wound healing, when immunity or other body functions of patient are compromised such as diabetes. Thus, there is a current need to develop some compound and formulations to treat such situations and cause faster wound closure. Wound contraction is an important component of wound healing. This involves pulling of surrounding skin circumferentially toward an open wound, which results in wound closure without formation of new tissues [30].

In our present wound healing experiment, all the animals remained healthy during the entire experiment. The feed as well as water intake and physical activity of all tested animals were normal. The topical applications of different formulations did not produce any undesirable effect like itching, hypersensitivity, edema, abscess etc. in any of the animal. The shedding of the scab was earlier in the nano $\mathrm{ZnO}$ treated wounds, as compared to other groups. The average time of shedding of scab in nano $\mathrm{ZnO}$ treated group was less than seven day, whereas it was around 8 10 days in other groups. The scab prevents the contraction of wound. In our present study, applications of ointment having $\mathrm{ZnO}$ nanoparticles caused the fast closure of the wounds. Gross photographs of different day's wounds of all the three groups are presented in Fig. 2a. Wound area in all three groups decreased in a time-dependent manner (Fig. 2b). However, percent wound contraction increased in a time-dependent manner in all the three groups. The results of wound area revealed that the wounds treated with $\mathrm{ZnO}$ nanoparticles have smaller wound area on respective days, as compared to other groups (Fig. 2a). These findings were further supported by the significantly increased percent wound contraction in $\mathrm{ZnO}$ nanoparticles treated group on different days, as compared to control and bulk $\mathrm{ZnO}$ treated groups (Fig. 2b). Additionally, bulk $\mathrm{ZnO}$ treated group, in comparison to control group, manifested some noticeable decreased wound area and increased percent wound contractions on various days. However, the extent of gross healing effect was more pronounced in the nano $\mathrm{ZnO}$ treated group. 


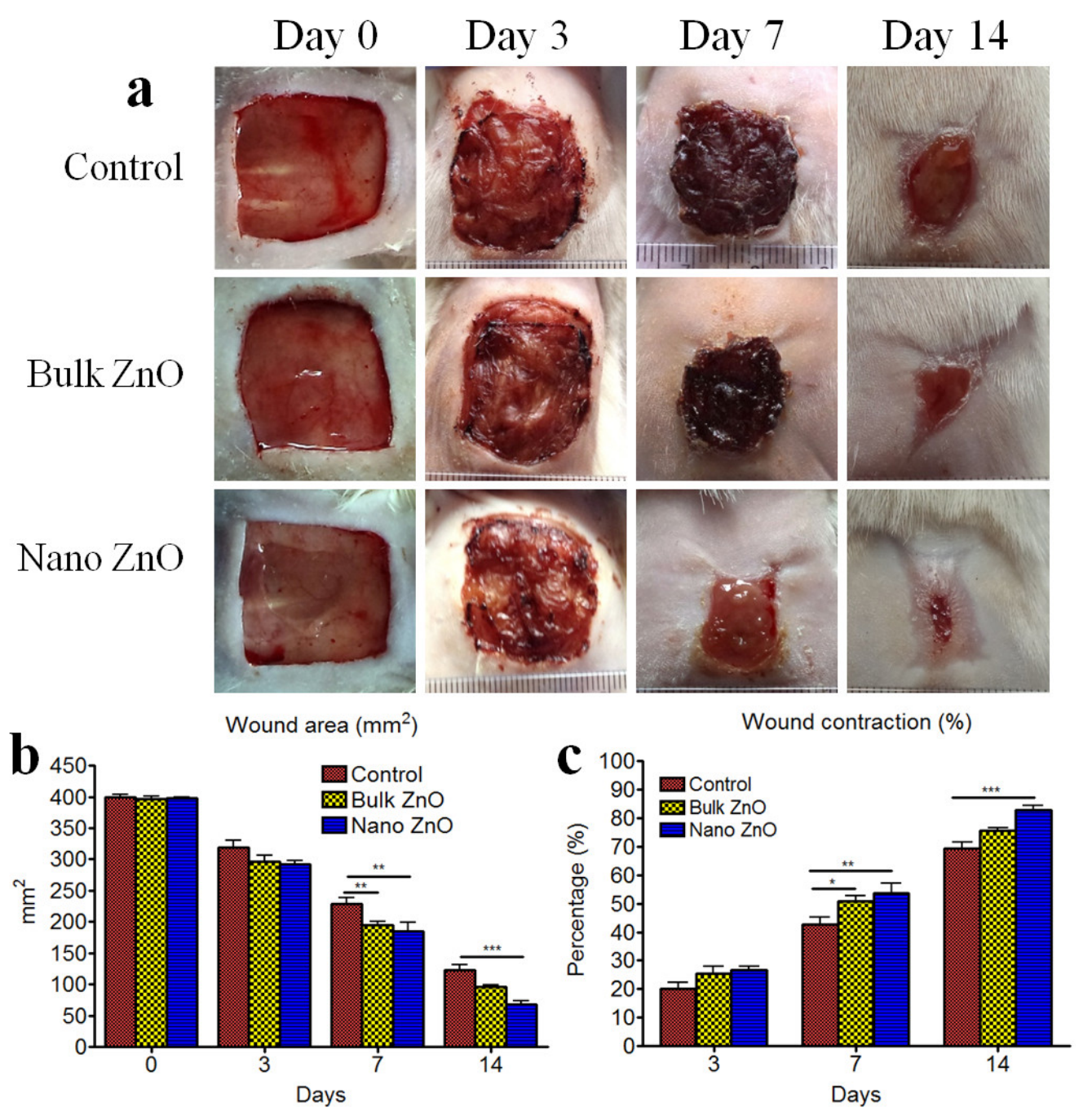

FIG. 2. (a) Gross photographs of wounds of different groups on days 0, 3, 7 and 14 post-wounding; (b \& c) Effects of topical application of nano $\mathrm{ZnO}$ on (b) wound area and (c) wound contraction in rats. Data are expressed as means \pm SEM. *, ** and *** represent $P<0.05, P<0.01$ and $P<0.001(n=5)$

As, the quality of healed tissue cannot be assessed grossly and sometime tissue showing faster healing on gross observations possesses poor quality and have the chances to get rupture easily. There are various important cells like monocytes, fibroblasts, and epithelial cells etc., which play vital roles for normal wound healing mechanisms. Thus, evaluation of the status of different important cell involved in the healing mechanism help to assess the quality of healing tissue. Therefore, on the last day of experiment, we performed histopathological studies of wound sections for microscopic assessment in order to evaluate the quality of healed tissue in different groups. In histopathological studies, the H\&E and Masson's Trichrome staining of the wound sections of different groups was done. The images in Fig. 3a and 3b represent the H\&E stained and Masson's Trichrome stained wound sections, respectively of the different groups. The Fig. 3c, 3d, 3e and 3f depict the semi-quantitative score of H\&E stained sections for inflammatory cells, blood vessels, epithelialization and wound maturity, respectively. During wound healing, fibroblasts cause production of extracellular matrix (ECM), which is primarily composed of collagen and fibronectin. This ECM provides the suitable scaffold on which granulation tissue formation, keratinocyte migration, and wound contraction occur. Collagen is considered as the major protein of the ECM, which provides wound strength and helps in homeostasis and epithelialization [31]. In present study, the H\&E stained sections of all the groups revealed well-formed granulation tissue with variation in proportions of inflammatory cells, fibroblast, blood vessels, collagen formation, extent of epithelialization etc. Several inflammatory cells may stimulate positive feedback mechanism to produce additional cytokines and growth factors at healing site and further play vital role in the proliferative phase of wound repair [32]. 
However, the inflammatory cells should not persist at wound site for longer durations because secretions of different inflammatory cytokines for longer time may decrease the wound healing. In the present study, mixed types of inflammatory cells were more evident in the control group, as compared to other groups. The inflammatory cells were less prevalent in the $\mathrm{ZnO}$ nanoparticle-treated group, which suggested the proper resolution of inflammatory phase in this group. The formation of blood vessels at wound site is necessary for delivery of important components (oxygen and nutrients), and removal of waste metabolites in order to attain proper healing process. Therefore, the healing process of wound gets delayed during impairment of angiogenesis. Angiogenesis starts as early as day 3 after wounding by endothelial migration, proliferation, and tube formation [33]. Thereafter, apoptosis (programmed cell death) of blood vessels starts to occur during the remodeling phase of healing, when there is not a significant requirement for nutrient supply is there, so that proper maturity of healing tissue can take place. In our present study, the numbers of blood vessels were more in control group, as compared to bulk and nano $\mathrm{ZnO}$ treated group. The fibroblast dominance was also more evident in the control group as compared to other groups. This revealed that nano $\mathrm{ZnO}$ treated wounds have attained better maturity than other treated wounds.

Further, the extracellular matrix provides the tensile strength to the healing wounds and collagen fibers are considered important components of this. The collagen fibers are delicate and loosely arranged during the earlier stages of wound healing. As the wound matures, collagen fibers attain organized pattern and compactness in thicker bundles [34]. In the present investigation, H\&E stained and Masson's Trichrome stained wound sections showed that collagen deposition was more in nano $\mathrm{ZnO}$ treated group. This group also showed significant organization and compactness of collagen fibers, as compared to control and bulk $\mathrm{ZnO}$ treated groups. Thick collagen bundles and wavy pattern of fibers revealed the maturity of collagen fibers in nano $\mathrm{ZnO}$ treated group, which was lacking in other groups. Moreover, the quantity and quality of collagen formation was better in the bulk $\mathrm{ZnO}$ treated group, as compared to the control group. The formation of an epithelial layer is also vital for wound contraction and proper wound healing. Epithelial layer formation reduces the distance between the wound margins and also protects the wound from outside so that proper haling can take place. Therefore, early formation of epithelial layer is beneficial for the efficient wound healing. In our present study, $\mathrm{ZnO}$ nanoparticle-based treatment of wounds resulted in the early regeneration of epithelial layer, which covered the more area of healing tissue. The other treatment groups only showed very partial formation of epithelial layer in few animals. Therefore, the score for epithelialization was more in nano $\mathrm{ZnO}$ treated group in our present study. Additionally, the overall wound maturity score was also higher in the $\mathrm{ZnO}$ nanoparticles treated group, as compared to control and bulk ZnO treated groups. The H\&E stained and Masson's Trichrome stained wound sections of last day of experiment are showing the dominance of fibroblasts, unorganized thin collagen fibers and a greater number of blood vessels in the control group, which revealed that healing process still has not entered in the remodeling phase. However, nano $\mathrm{ZnO}$ treated wounds have fewer fibroblast numbers, fewer blood vessels, thick collagen fibers, early formation of epithelial layer etc. All these are the indicators of entry of the healing process in the remodeling phase in nano $\mathrm{ZnO}$ treated group. It has been well reported previously that $\mathrm{ZnO}$ application on wounds accelerates the healing of acute and chronic wounds and it also possesses antibacterial, antifungal, cytoprotactive, and anti-inflammatory properties, which make it a suitable topical wound healing agent [35-37]. Its use for the treatment of various dermatitis, diaper rashes, diaper wipes, blisters, and open skin sores hasbeen also documented [38, 39].

In the last few years, different types of nanoparticles have been synthesized by using several methods; these nanoparticles have shown promising wound healing potentials even in comparison to the standard treatments as well as earlier used their bulk forms. In biomedical fields, the intervention of nanotechnology for any material significantly influences its size, shape, electrical charge, biochemical features such as hydrophobicity, delivery, interaction with biological targets, deeper levels of tissue penetration (either directly through cell membranes or through phagocytosis) and cellular responses (receptor recognition), which can significantly affect the healing processes of any type of wound [40-43]. In a previous study, silver nanoparticles accelerated the wound healing rate by increased wound contraction, increased proliferation and migration of keratinocytes along with a differentiation of fibroblasts into myofibroblasts [44]. Wound healing has been promoted by silica-gold core-shell nanoparticles due to potent anti-inflammatory and antioxidant properties of gold nanoparticles [45]. Moreover, silver nanoparticles application on porcine wounds significantly increased apoptosis of the inflammatory cells present in the dermis, decreased levels of pro-inflammatory cytokines (TNF- $\alpha$ and IL-8) and increased the levels of anti-inflammatory cytokines (IL-4, EGF, KGF, and KGF-2) [46]. Several other nanoparticles of titanium oxide [47], curcumin [48], and copper [49] have also shown significant immunomodulatory effects. The combination preparation having gold nanoparticles, epigallocatechin gallate, and $\alpha$-lipoic acid significantly accelerated diabetic cutaneous wound healing through angiogenesis regulation and antiinflammatory effects, and immunoblotting results of this study revealed increased expression of VEGF [50]. Collagen deposition, their structural alignment and tensile strength have been improved by the administration of silver nanoparticles in the healing tissue of cutaneous wounds [51,52]. These several studies have clearly 


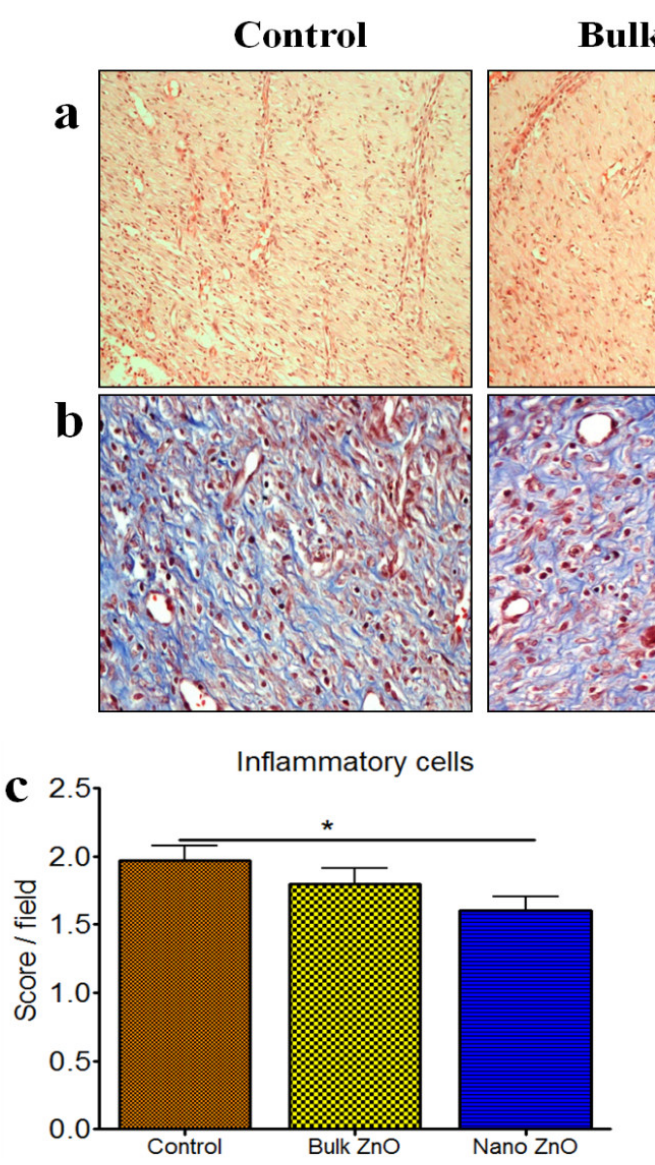

Bulk ZnO

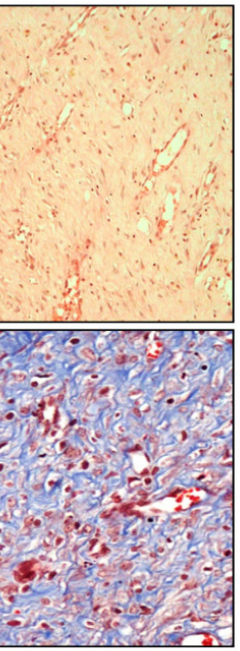

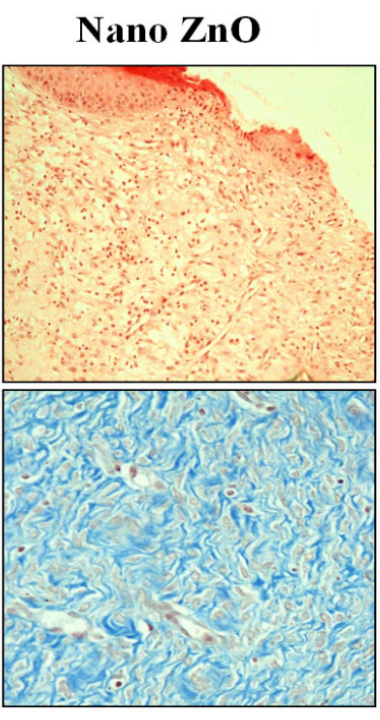

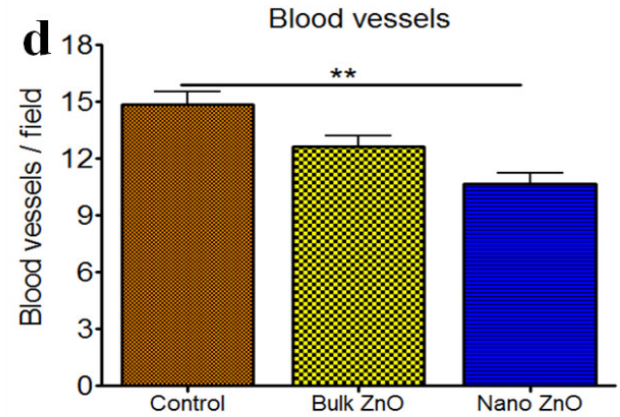

f

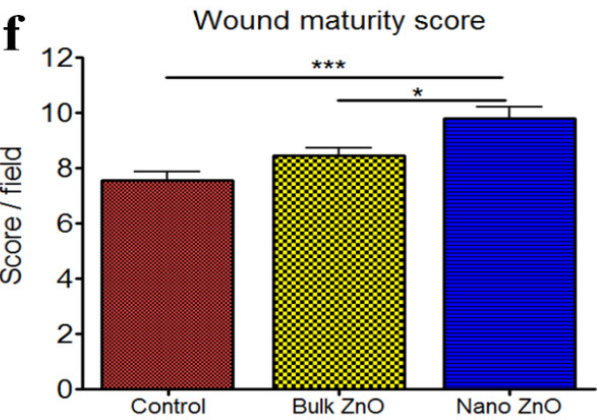

FIG. 3. Representative images of (a) haematoxylin\& eosin (H \& E) (20×) stained and (b) Masson's Trichrome $(40 \times)$ stained wound sections of different treatment groups. Semi-quantitative score of (c) inflammatory cells, (d) blood vessels density, (e) epithelialization and (f) wound maturity in $\mathrm{H}$ $\&$ E stained cutaneous wound sections of different treatment groups. Data are expressed as mean \pm SEM. * and *** represent $P<0.05$ and $P<0.001$

explained that the mechanism of healing is very complex and there is involvement of several cytokine, growth factors, enzymes, cells etc., which markedly influence the outcome of healing process. Moreover, there is not a single pathway by which different metals or metal oxides or other nanoparticles promote the healing mechanisms. The exact mechanism of healing by $\mathrm{ZnO}$ is also not known. Apart from diverse pharmacological properties of $\mathrm{ZnO}$, it has been shown to increase the secretion of fibroblast growth factor (FGF), suggesting that zinc in granulating wounds is also possibly capable of up-regulating growth factors [53]. The insulin-like growth factor-I (IGF-I) is vital for homeostasis of epidermis and the zinc-dependent matrix metalloproteinases (MMPs) are required for optimal epithelial migration [54,55]. Further, $\mathrm{ZnO}$ augments the endogenous expression of IGF-I in granulation tissue and can also activate zinc-dependent MMPs to facilitate the migration of keratinocytes [56]. Some researchers have suggested that local applications of $\mathrm{ZnO}$ accelerates wound healing by its anti-bacterial, anti-inflammatory, increase reepithelization and 
activation of metalloproteinase enzymes properties $[57,58]$. Decreasing the dimension of nanoparticles has a pronounced effect on their physical properties, which significantly differ from those of the bulk material. Therefore, in our present study, the better wound healing potential of nano $\mathrm{ZnO}$ in comparison to its bulk form could be due the novel and distinct physical, chemical, and biological properties and functionality due to their nanoscale size. Thus, in the present study, the increased healing potential of nano form of $\mathrm{ZnO}$ in comparison to its bulk form could also be due to the efficient modulation of several cytokines, growth factors, enzymes, cells etc involved in different phases of healing. The potent anti-inflammatory action of nano $\mathrm{ZnO}$ may be responsible for faster completion of inflammatory phase and resulting in early entry of wound to proliferative phase. In our present study, nano $\mathrm{ZnO}(2 \%)$ showed better wound healing potential than bulk $\mathrm{ZnO}(20 \%)$ even at ten-fold lower concentrations. Thus, nano $\mathrm{ZnO}$, as a promising wound healing agent, can be used for the treatment of different types of wounds. However, some additional studies like healing effect in other species, healing effect in complicated wounds (burn wounds, diabetic wounds etc.), local irritation studies, biocompatibility, toxicity studies, calculation of lethal dose 50 (LD50), stability of nano $\mathrm{ZnO}$ formulation, healing effect of lower concentrations of nano $\mathrm{ZnO}$ etc. may be required to be conducted before proceeding to clinical studies.

\section{Conclusion}

On the basis of results obtained for the present study, it might be concluded that topical application of $\mathrm{ZnO}$ nanoparticles $(2 \%)$ in ointment base caused faster wound healing and the healing was better than bulk $\mathrm{ZnO}$ treatment. Thus, nano $\mathrm{ZnO}$ might be envisioned as a promising healing agent in future.

\section{References}

[1] Bello D., Martin J., et al. Physicochemical and morphological characterisation of nanoparticles from photocopiers: implications for environmental health. Nanotoxicology, 2012, 7 (5), P. 989-1003.

[2] Demokritou P., Gass S., et al. An in vivo and in vitro toxicological characterisation of realistic nanoscale $\mathrm{CeO}_{2}$ inhalation exposures. Nanotoxicology, 2013, 7 (8), P. 1338-1350.

[3] Pirela S., Molina R., et al. Effects of copy center particles on the lungs: a toxicological characterization using a Balb/c mouse model. Inhal. Toxicol., 2013, 25 (9), P. 498-508.

[4] Larsen S.T., Roursgaard M., Jensen K.A., Nielsen G.D. Nano titanium dioxide particles promote allergic sensitization and lung inflammation in mice. Basic Clin. Pharmacol. Toxicol., 2010, 106 (2), P. 114-117.

[5] Li J.J.E., Muralikrishnan S., et al. Nanoparticle-induced pulmonary toxicity. Exp. Biol. Med., 2010, 235 (9), P. $1025-1033$.

[6] Cohen J., Deloid G., Pyrgiotakis G., Demokritou P. Interactions of engineered nanomaterials in physiological media and implications for in vitro dosimetry. Nanotoxicology, 2013, 7 (4), P. 417-431.

[7] Garcia-Orue I., Gainza G., et al. Nanotechnology approaches for skin wound regeneration using drugdelivery systems. Nanobiomaterials in Soft Tissue Engineering, 2016, P. 31-55.

[8] Mishra P.K., Mishra H., et al. Zinc oxide nanoparticles: a promising nanomaterial for biomedical applications. Drug Discovery Today, 2017, 22 (12), P. 1825-1834.

[9] Akila S., Nanda A. In-Vivo Wound Healing Activity of Silver Nanoparticles: An Investigation. Int. J. Sci. Res., 2014, 3 (7), P. 1208-1212.

[10] Alizadeh S., Seyedalipour B., et al. Copper nanoparticles promote rapid wound healing in acute full thickness defect via acceleration of skin cell migration, proliferation, and neovascularization. Biochem. Biophys. Res. Commun., 2019,517 (4), P. $684-690$.

[11] Tao B., Lin C., et al. Copper-nanoparticle-embedded hydrogel for killing bacteria and promoting wound healing with photothermal therapy. $J$. Mater. Chem. B, 2019, 7 (15), P. 2534-2548.

[12] Ziv-Polat O., Topaz M., Brosh T., Margel S. Enhancement of incisional wound healing by thrombin conjugated iron oxide nanoparticles. Biomaterials, 2010, 31 (4), P. 741-747.

[13] Hamam F., Nasr A. Curcumin-loaded mesoporous silica particles as wound-healing agent: An in vivo study. Saudi J. Med. Med. Sci., 2020, 8 (1), P. 17-24.

[14] Kheradmand F., Mousavi A., Nurmohamadi E. Zinc and molecular mechanisms involved in its homeostasis. J. Paramed. Faculty, 2009, 4 (1), P. 34-38.

[15] Jones N., Ray B., Ranjit K.T., Manna A.C. Antibacterial activity of ZnO nanoparticle suspensions on a broad spectrum of microorganisms. FEMS Microbiol. Lett., 2008, 279, P. 71-76.

[16] Wang S.Q., Tooley I.R. Photoprotection in the era of nanotechnology. Semin. Cutan. Med. Surg., 2011, 30, P. $210-213$.

[17] Prasad A.S., Oberleas D. Thymidine kinase activity and incorporation of thymidine into DNA in zinc-deficient tissue. J. Lab. Clin. Med., 1974, 83, P. 634-639.

[18] Sandstead H.H., Lanier V.C., Shepard G.H., Gillespie D.D. Effects of zinc deficiency and zinc supplementation. Amer. J. Clin. Nutr., 1970, 23, P. 514-519.

[19] Prasad A.S. Zinc: an overview. Nutr., 1995, 11, P. 93-99.

[20] Agren M.S., Chvapil M., Franzén L. Enhancement of re-epithellalization with topical zinc oxide in porcine partialthickness wounds. J. Surg. Res., 1991, 50, P. 101-105

[21] Kaushik M., Niranjan R., et al. Investigations on the antimicrobial activity and wound healing potential of ZnO nanoparticles. Appl. Surf. Sci., 2019, 479, P. 1169-1177.

[22] Rezende C.P., da Silva J.B., Mohallem N.D.S. Influence of drying on the characteristics of zinc oxide nanoparticles. Braz. J. Phys., 2009, 39, P. 248-251. 
[23] Hajiaghaalipour F., Kanthimathi M.S., Abdulla M.A., Sanusi J. The effect of Camellia sinensis on wound healing potential in an animal model. Evid. Based Compl. Alt. Med., 2013, 386734.

[24] Abramov Y., Golden B., et al. Histologic characterization of vaginal vs. abdominal surgical wound healing in a rabbit model. Wound Repair Regen., 2007, 15 (1), P. 80-86.

[25] Greenhalgh D.G., Sprugel K.H., Murray M.J., Ross, R. PDGF and FGF stimulate healing in the genetically diabetic mouse. Am. J. Pathol., 1990, 136, P. 1235-1246.

[26] Kant V., Kumar D., et al. Topical application of substance P promotes wound healing in streptozotocin-induced diabetic rats. Cytokine, 2015, 73, P. 144-155.

[27] Cho K., Wang X., Nie S., Shin D.M. Therapeutic nanoparticles for drug delivery in cancer. Clin, Cancer, Res., 2008, 14, P. $1310-1316$.

[28] Fine N., Mustoe T. Wound Healing. Philadelphia: Lippincott Williams \& Wilkins, 2006.

[29] Wertheimer E. Diabetic skin complications: a need for reorganizing the categories of diabetes-associated complications. Isr. Med. Assoc. J., 2004, 6, P. 287-289.

[30] Bae S.H., Bae Y.C., Nam S.B., Choi S.J. A Skin Fixation Method For Decreasing The Influence Of Wound Contraction On Wound Healing In A Rat Model. Arch. Plast. Surg., 2012, 39 (5), P. 457-462.

[31] Singer A.J., Clark R.A.F. Cutaneous wound healing. N. Engl. J. Med., 1999, 341, P. 738-746.

[32] Werner R., Grose S. Regulation of wound healing by growth factors and cytokines. Physiol. Rev., 2003, 83, P. 835-870.

[33] Brem H., Folkman J. Angiogenesis and basic fibroblast growth factor during wound healing. In: Friedlander G.E., Lane J.M., (ed.) Bone Formation and Repair, Rosemont: American Academy of Orthopedic Surgeons, 1994, P. 213-222.

[34] Pereira M.C., Pinho C.B., et al. Influence of $670 \mathrm{~nm}$ low-level laser therapy on mast cells and vascular response of cutaneous injuries. $J$. Photochem. Photobiol. B, 2010, 98, P. 188-192.

[35] Selvam S., Rajiv Gandhi R., et al. Antibacterial effect of novel synthesized sulfated cyclodextrin crosslinkedcotton fabric and its improved antibacterial activities with $\mathrm{ZnO}, \mathrm{TiO}_{2}$ and $\mathrm{Ag}$ nanoparticles coating. Int. J. Pharm., 2012, 434 (1-2), P. 366-374.

[36] Subhasree R.S., Selvakumar D., Kumar N.S. Hydrothermal mediated synthe-sis of ZnO nanorods and their antibacterial properties. Lett. Appl. NanoBioSci., 2012, 1, P. 2-7.

[37] Vlad S., Tanase C., et al. Antifungal behaviour of polyurethane membranes with zincoxide nanoparticles. Digest J. Nanomater. Biostruct., 2012, 7, P. 51-58.

[38] Kumar P.T.S., Lakshmanan V.K., et al. Flexible and microporous chitosanhydrogel/nanoZnO composite bandages for wound dressing: in vitro and in vivoevaluation. ACS Appl. Mater. Interfaces., 2012, 4, P. 2618-2629.

[39] Shalumon K.T., Anulekha K.H., et al. Sodium alginate/poly vinyl alcohol/nano ZnO composite nanofibers forantibacterial wound dressings. Int. J. Biol. Macromol., 2011, 49, P. 247-254.

[40] Hamdan S., Pastar I., et al. Nanotechnology-Driven Therapeutic Interventions in Wound Healing: Potential Uses and Applications. ACS Cent. Sci., 2017, 3, P. 163-175.

[41] Lin P.C., Lin S., Wang P.C., Sridhar R. Techniques for physicochemical characterization of nanomaterials. Biotechnol. Adv., 2014, 32, P. 711726.

[42] Ferrari M. Nanogeometry: Beyond drug delivery. Nat. Nanotechnol., 2008, 3, P. 131-132.

[43] George S., Lin S., et al. Surface defects on plate-shaped silver nanoparticles contribute to its hazard potential in a fish gill cell line and zebrafish embryos. ACS Nano, 2012, 6, P. 3745-3759.

[44] Liu X., Lee P.Y., et al. Silver nanoparticles mediate differential responses in keratinocytes and fibroblasts during skin wound healing. Chem. Med. Chem., 2010, 5 (3), P. 468-475.

[45] Li X., Wang H., et al. Effect of composite SiO(2) @ AuNPs on wound healing: in vitro and vivo studies. J. Colloid. Interface Sci., 2015, 445, P. 312-319.

[46] Nadworny P.L., Landry B.K., et al. Does nanocrystalline silver have a transferable effect? Wound Repair Regen., 2010, 18, P. $254-265$.

[47] Archana D., Dutta J., Dutta P.K. Evaluation of chitosan nano dressing for wound healing: characterization, in vitro and in vivo studies. Int. J. Biol. Macromol., 2013, 57, P. 193-203.

[48] Chereddy K.K., Coco R., et al. Combined effect of PLGA and curcumin on wound healing activity. J. Control Release, 2013, 171, P. 208-215.

[49] Tiwari M., Narayankan K., et al. Biosynthesis and wound healing activity of copper nanoparticles. IET Nanobiotechnol., 2014, 8, P. $230-237$.

[50] Chen S.A., Chen H.M., et al. Topical treatment with antioxidants and Au nanoparticles promote healing of diabetic wound through receptor for advance glycation end-products. Eur. J. Pharm. Sci., 2012, 47, P. 875-883.

[51] Guthrie K.M., Agarwal A., et al. Integration of silver nanoparticle-impregnated polyelectrolyte multilayers into murine-splinted cutaneous wound beds. J. Burn Care Res., 2013, 34, P. 359-367.

[52] Kwan K.H., Liu X., et al. Modulation of collagen alignment by silver nanoparticles results in better mechanical properties in wound healing. Nanomed., 2011, 7, P. 497-504.

[53] Baroni A., Perfetto B., et al. Topical amikacin formulation induces fibroblast growth factorand cytokine releasefrom human dermal fibroblasts. Arch. Dermatol. Res., 1999, 291, P. 296-299.

[54] Tarnow P., Agren M., Steenfos H., Jansson J.O. Topical zinc oxide treatment increases endogenous gene expression of insulin-like growth factor-I (IGF-1) in granulation tissue from porcine wounds. Scand. J. Plast. Reconstr. Surg. Hand. Surg., 1994, 28, P. 255-259.

[55] Agren M.S. Matrix metalloproteinases (MMPs) are required for re-epithelialization of cutaneous wounds. Arch. Dermatol. Res., 1999, 291, P. 583-590.

[56] Ägren M.S., Steenfos H.H., Tarnow P., Jansson J. Auguments endogenous expression of insulin-like growth factor-I (IGF-I) and activates matrix metalloproteinases (MMPs) in wounds. EWMA J., 2001, 138169909.

[57] Lansdown A.B., Mirastschijski U., et al. Zinc in wound healing: theoretical, experimental, and clinical aspects. Wound Repair Regen., 2007, 15 (1), P. 2-16.

[58] Iwata M., Takebayashi T., et al. Zinc accumulation and metallothionein gene expression in the proliferating epidermis during wound healing in mouse skin. Histochem. Cell. Biol., 1999, 112 (4), P. 283-290. 